\title{
Cyclotron line energy in Hercules X-1: stable after the decay
}

\author{
R. Staubert ${ }^{1}$, L. Ducci ${ }^{1}$, L. Ji ${ }^{1}$, F. Fürst ${ }^{2}$, J. Wilms $^{3}$, R. E. Rothschild ${ }^{4}$, K. Pottschmidt ${ }^{5,6}$, \\ M. Brumback ${ }^{7}$, and F. Harrison ${ }^{8}$
}

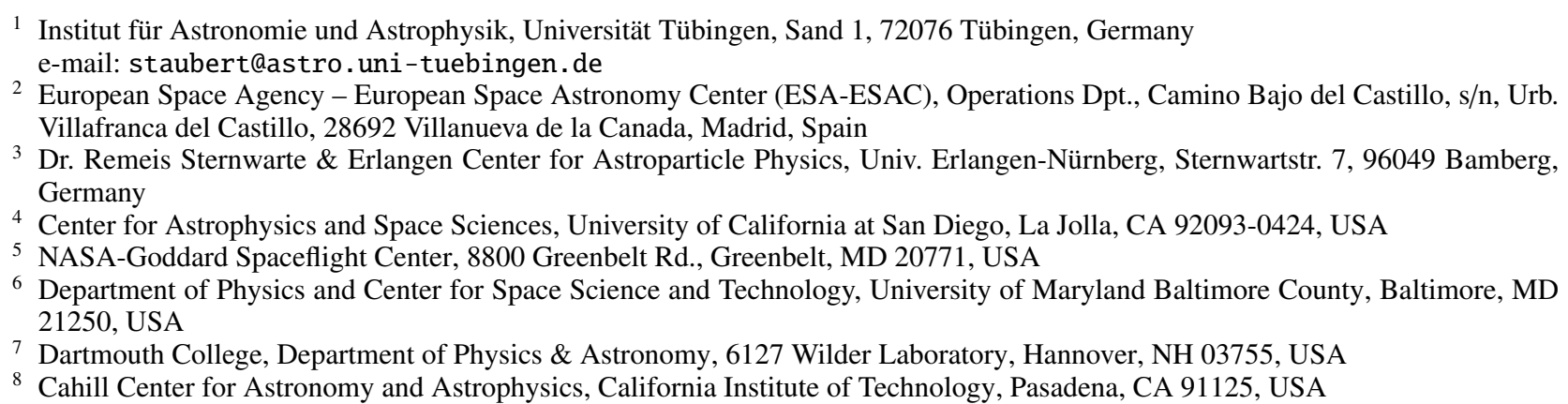

Received 6 July 2020 / Accepted 14 August 2020

\begin{abstract}
We summarize the results of a dedicated effort made between 2012 and 2019 to follow the evolution of the cyclotron line in Her X-1 through repeated NUSTAR observations. The previously observed nearly 20-year-long decay of the cyclotron line energy has ended in 2012: from then on, the pulse-phase-averaged flux-corrected cyclotron line energy has remained stable and constant at an average value of $E_{\mathrm{cyc}}=(37.44 \pm 0.07) \mathrm{keV}$ (normalized to a flux level of $\left.6.8 \mathrm{RXTE} / \mathrm{ASM}-\mathrm{cts} \mathrm{s}^{-1}\right)$. The flux dependence of $E_{\text {cyc }}$ discovered in 2007 is now measured with high precision, giving a slope of $(0.675 \pm 0.075) \mathrm{keV} /\left(\mathrm{ASM}-\mathrm{cts} \mathrm{s}^{-1}\right)$, corresponding to an increase of $6.5 \%$ of $E_{\text {cyc }}$ for an increase in flux by a factor of two. We also find that all line parameters as well as the continuum parameters show a correlation with X-ray flux. While a correlation between $E_{\text {cyc }}$ and X-ray flux (both positive and negative) is now known for several accreting binaries with various suggestions for the underlying physics, the phenomenon of a long-term decay has so far only been seen in Her X-1 and Vela X-1, with far less convincing explanations.
\end{abstract}

Key words. stars: magnetic field - radiation mechanisms: general - scattering - pulsars: individual: Her X-1 - stars: neutron X-rays: stars

\section{Introduction}

The eclipsing binary Her X-1/HZ Her is a low-mass X-ray binary (LMXB) that was discovered as an X-ray source by the first X-ray satellite UHURU in 1971 (Tananbaum et al. 1972). Similar to Cen X-3, the source was identified as an X-ray pulsar, powered by mass accretion from its companion. Her X-1 is one of the most interesting X-ray pulsars because its observable features vary widely. This source has been introduced many times; we refer to some of the most recent introductions, for example, Staubert et al. (2017, 2019) and Sazonov et al. (2020). In order to maintain some degree of completeness within this contribution, we list the following main features of Her X-1: the spin period of the neutron star is $1.24 \mathrm{~s}$, the orbital period is $1.7 \mathrm{~d}$ (identified by eclipses and the modulation in the pulse arrival times), the superorbital flux is modulated with a somewhat variable period of $\sim 35 \mathrm{~d}$. This on-off variation can be understood as being due to the precession of a warped accretion disk (Petterson 1977; Schandl \& Meyer 1994). Because the binary is highly inclined $\left(i>80^{\circ}\right)$, we see the disk nearly edgeon (Gerend \& Boynton 1976). The precessing warped disk covers the central X-ray source during a substantial portion of the $35 \mathrm{~d}$ period (Klochkov et al. 2006, 2008).
The X-ray spectrum Her X-1 is a power-law continuum with exponential cutoff, as is typical of accreting binary pulsars (Wolff et al. 2016). The cyclotron line around $37 \mathrm{keV}$, discovered in a balloon observation in 1976 (Trümper et al. 1978), is due to resonant scattering of photons by electrons on Landau levels in the $\sim 10^{12}$ Gauss magnetic field at the polar caps of the neutron star. It is therefore often referred to as a cyclotron resonant scattering feature (CRSF). The energy spacing between the Landau levels is approximately given by $E_{\text {cyc }} \approx 11.6 \mathrm{keV} B_{12}$, where $B_{12}$ is the magnetic field strength in units of $10^{12}$ Gauss. When the gravitational redshift is taken into account, the magnetic field strength at the site of the emission of the X-ray spectrum can be measured directly from the observed energy of the fundamental cyclotron line in the X-ray spectrum: $B_{12} \approx(1+z) E_{\mathrm{obs}} / 11.6 \mathrm{keV}$, where $z$ is the gravitation redshift (Schwarm et al. 2017).

The discovery of the cyclotron feature in the spectrum of Her X-1 was the first direct measurement of the surface magnetic field strength of a neutron star. In contrast to other ways of estimating such a magnetic field strength, no further model assumptions are needed. We now know about 35 binary X-ray pulsars that show cyclotron lines in their spectra, generally between a few $\mathrm{keV}$ and $\sim 100 \mathrm{keV}$ (for reviews, see Staubert et al. 2019; Revnivtsev \& Mereghetti 2016; Caballero \& Wilms 2012; 
Table 1. Details on NUSTAR observations of Her X-1 between 2012 and 2020.

\begin{tabular}{|c|c|c|c|c|c|c|c|c|}
\hline $\begin{array}{l}\text { Observation } \\
\text { date }\end{array}$ & Obs ID & $\begin{array}{l}\text { 35-day } \\
\text { cycle } \\
\text { no. }{ }^{(a)}\end{array}$ & $\begin{array}{l}\begin{array}{l}\text { Start } \\
\text { of obs }\end{array} \\
\text { [MJD] }\end{array}$ & $\begin{array}{l}\text { End } \\
\text { of obs } \\
\text { [MJD] }\end{array}$ & $\begin{array}{l}\begin{array}{l}\text { Center } \\
\text { of obs }\end{array} \\
\text { [MJD] }\end{array}$ & $\begin{array}{l}\text { Net } \\
\text { expo } \\
\text { sure } \\
{[\mathrm{ksec}]}\end{array}$ & $\begin{array}{l}\text { 35-day } \\
\text { Turn-On }{ }^{(b)} \\
\text { [MJD] }\end{array}$ & $\begin{array}{l}\text { 35-day } \\
\text { phase }{ }^{(c)} \\
\text { of center } \\
\text { of obs }\end{array}$ \\
\hline 22 & 6005 & 427 & 56192.19 & 56192.77 & 56192.48 & $\sim 22$ & 56189. & 0.100 \\
\hline & & & & & & & & \\
\hline & & & & & 3 & & & 1 \\
\hline $05 \mathrm{Auq}$ & 30302012002 & & & & 57970.81 & 28 & & 0.147 \\
\hline & & & & & & & & \\
\hline 1 & 30 & 49 & & & & 28.4 & 0.3 & 0.044 \\
\hline $09 \mathrm{Feb} 2019$ & 30402034002 & 494 & 58523.41 & 58523.85 & 58523.63 & 18.3 & $58516.6 \pm 0.2$ & $0.202^{(e)}$ \\
\hline & & & & & & & & \\
\hline 23 Jun 2019 & 30402009004 & 498 & 58657.34 & 58658.06 & 58657.70 & 27.1 & $58654.1 \pm 0.2$ & 0.102 \\
\hline
\end{tabular}

Notes. ${ }^{(a)} 35$-day cycle numbering is according to Staubert et al. (1983); ${ }^{(b)}$ as determined from the monitoring data of Swift/BAT; ${ }^{(c)}$ using $P_{35}=34.85 \mathrm{~d}$; ${ }^{(d)}$ see Fürst et al. (2013), Table 1 ; $^{(e)}$ this observation is at a particularly high 35 -day phase.

Wilms 2012; Terada et al. 2007; Heindl et al. 2004; Staubert 2003; Coburn et al. 2002).

Significant variability has been observed with the CRSF in Her X-1 regarding its centroid energy $E_{\mathrm{cyc}}$ and other characteristic parameters such as its width and optical depth. These parameters vary with pulse phase, with luminosity, and with time (Staubert et al. 2014). Her X-1 was the source in which all these variations were observed for the first time: a positive correlation between $E_{\mathrm{cyc}}$ and the X-ray luminosity $L_{\mathrm{X}}$ (Staubert et al. 2007) (confirmed on short timescales by the pulse-amplitude-resolved technique by Klochkov et al. 2011), and a long-term decay of $E_{\mathrm{cyc}}$, coexisting with the luminosity dependence (Staubert et al. 2014, 2016). The long-term decay was confirmed by Klochkov et al. (2015) using monitoring data of Swift $/ \mathrm{BAT}^{1}$. For the current knowledge about such variations in other accreting X-ray pulsars, see Staubert et al. (2019).

The long-term decay has been of particular interest, as has the question whether this would end at some time or even become inverted, such that $E_{\text {cyc }}$ would rise again. This appeared to have been observed in 2017 (Staubert et al. 2017). We show here, however, that the decay did end, but a turn-up has not been observed (see Sect. 3.1). The end of the decay is supported through observations with Swift/BAT (Ji et al. 2019), InsightHXMT (Xiao et al. 2019) and Astrosat (Bala et al. 2020).

Here we summarize the results of nine observations of Her X-1 by $N u S T A R^{2}$ in 2012 to 2019 with regard to the cyclotron resonance scattering feature in the pulse-averaged X-ray spectrum: The CRSF energy has apparently stopped its $\sim 20$-year-long decay and has been constant approximately since 2012. In addition to the CRSF centroid energy, its width and strength are clearly also correlated with flux, and the dependences are now measured with high precision. We further present evidence for a dependence of all continuum parameters on X-ray flux.

\section{Observations and analysis}

In Table 1 we list nine observations of Her X-1 performed by NuSTAR (Harrison et al. 2013) in 2012 to 2019. They were made

\footnotetext{
1 BAT refers to the Burst Alert Telescope on the NASA mission Swift.

2 NuSTAR refers to the NASA mission Nuclear Spectroscopic Telescope Array.
}

close to the maximum flux of a main-on state. We also list the net exposure times (which vary between $4.3 \mathrm{ks}$ and $36.6 \mathrm{ks}$ ), the times of the respective 35-day turn-on, and the 35-day phase of the center of the respective observations. The details of the data analysis are similar to those described by Staubert et al. (2014, 2016). We used the standard nupipeline and nuproducts utilities (01 Apr 20_v1.9.2) and XSPEC ${ }^{3}$ v12.11 as part of HEA$\mathrm{SOFT}^{4}$. The source extraction diameter was selected between $90 \operatorname{arcsec}$ and $120 \operatorname{arcsec}$ depending on the brightness of the source. All values given are from simultaneous spectral fitting of the data from both focal plane detectors, unless otherwise stated. The spectral function used for all observations was the XSPEC function highecut in combination with a power law,

$I_{\mathrm{E}}= \begin{cases}K \cdot E^{-\Gamma}, & \text { if } E \leq E_{\text {cut }} \\ K \cdot E^{-\Gamma} \exp \left(-\frac{E-E_{\text {cut }}}{E_{\text {fold }}}\right), & \text { if } E>E_{\text {cut }}\end{cases}$

Here $\Gamma$ is the power-law (photon) index, $E_{\text {cut }}$ is the energy at which the cutoff sets in, and $E_{\text {fold }}$ is the $e$-folding energy describing the flux decay. The function contains a discontinuity of its first derivative (a break) at $E=E_{\text {cut }}$. In order to smooth this break, an artificial small Gaussian absorption line was generally added, with the center energy fixed to $E_{\text {cut }}$ and a free width and depth. Neither the power law nor the exponential cutoff are affected by this (e.g., Coburn et al. 2002).

The cyclotron line is modeled by the Gaussian-shaped gabs function: To model the cyclotron line, the continuum functions described above are modeled by the inclusion of a corresponding multiplicative component of the form $e^{-\tau(E)}$, where the optical depth $\tau(E)$ has a Gaussian profile,

$\tau(E)=\tau_{0} \exp \left[-\frac{\left(E-E_{\mathrm{cyc}}\right)^{2}}{2 \sigma_{\mathrm{cyc}}^{2}}\right]$

with $\tau_{0}, E_{\mathrm{cyc}}$, and $\sigma_{\mathrm{cyc}}$ being the central optical depth, the centroid energy, and the width of the line. We note that in the popular XSPEC realization of this function gabs, $\tau_{0}$ is not explicitly used as a fit parameter. Instead, a product $\tau_{0} \sqrt{2 \pi} \sigma_{\text {cyc }}$ is defined as the strength of the line.

\footnotetext{
3 https://heasarc.gsfc.nasa.gov/xanadu/xspec/

4 https://heasarc.gsfc.nasa.gov/lheasoft/, 6.27.2, caldb release 20191219.
} 
Table 2. Summary of the spectral analysis of nine NUSTAR observations of Her X-1.

\begin{tabular}{|c|c|c|c|c|c|c|c|c|}
\hline $\begin{array}{l}35 \mathrm{~d} \\
\text { cycle } \\
\text { no. }(e)\end{array}$ & $\begin{array}{l}\text { Max. flux } \\
\text { of } 35 \mathrm{~d} \\
\text { cycle } \\
{\left[\text { ASM-cts s }{ }^{-1} \text { ] }\right.}\end{array}$ & $\begin{array}{l}\text { Observed } \\
\text { line } \\
\text { energy } \\
{[\mathrm{keV}]}\end{array}$ & $\begin{array}{l}\text { Line } \\
\text { width } \\
\sigma \\
{[\mathrm{keV}]} \\
\end{array}$ & $\begin{array}{l}\begin{array}{l}\text { Line } \\
\text { strength }\end{array}{ }^{(a)} \\
{[\mathrm{keV}]}\end{array}$ & $\begin{array}{l}E_{\mathrm{cyc}} \\
\text { norm. to } 6.8 \\
\text { ASM-cts s } \\
{[\mathrm{keV}]} \\
\end{array}$ & {$[\mathrm{keV}]$} & $E_{\text {fold }}$ & $\begin{array}{l}\text { Power- } \\
\text { law } \\
\text { index } \\
\Gamma \\
\end{array}$ \\
\hline 427 & $6.60 \pm 0.37$ & $37.40 \pm 0.25^{(b)}$ & $5.76 \pm 0.29$ & $8.86 \pm 0.87$ & $37.54 \pm 0.25$ & $20.68 \pm 0.27$ & $9.95 \pm 0.13$ & $0.920 \pm 0.004$ \\
\hline 457 & $2.96 \pm 0.20$ & $34.79 \pm$ & $4.46 \pm 0.22$ & $4.70 \pm$ & $37.38 \pm$ & $19.86=$ & $9.37 \pm 0.09$ & $0.929 \pm 0.003$ \\
\hline 468 & $6.50 \pm 0.20$ & $37.18=$ & $7 \pm$ & $8.83 \pm$ & 37.38 & 20.86 & 10.1 & 0.985 \\
\hline 478 & $4.10 \pm$ & 18 & $.94 \pm$ & $5.90 \pm$ & 37.44 & 19.98 & $9.79=$ & 0.002 \\
\hline 484 & $4.09 \pm$ & $35.67 \pm$ & $84 \pm$ & $6.10 \pm$ & 37.50 & 20.04 & 10.16 & $0.963 \pm 0.002$ \\
\hline 490 & $5.60 \pm 0$ & $36.65 \pm$ & $5.61 \pm$ & $8.44 \pm$ & 37.46 & $20.45 \pm$ & $9.79 \pm$ & $0.974 \pm 0.002$ \\
\hline 494 & $5.02 \pm 0.46$ & $36.28 \pm 0.22$ & $5.26 \pm 0.24$ & $7.21 \pm 0.53$ & $37.48 \pm 0.23$ & $19.56 \pm 0.12$ & $9.65 \pm 0.11$ & $0.885 \pm 0.002^{(f)}$ \\
\hline 495 & $3.72 \pm 0.56$ & $35.36 \pm 0.41$ & $4.76 \pm 0.45$ & $6.38 \pm 0.98$ & $37.44 \pm 0.43$ & $19.62 \pm 0.29$ & $9.49 \pm 0.24$ & $0.934 \pm 0.005$ \\
\hline $498^{(d)}$ & $4.00 \pm 0.37$ & $35.65 \pm 0.21$ & $5.01 \pm 0.25$ & $7.02 \pm 0.50$ & $37.54 \pm 0.22$ & $19.81 \pm 0.16$ & $9.38 \pm 0.09$ & $0.932 \pm 0.001$ \\
\hline
\end{tabular}

Notes. The spectral parameters were found by applying the XSPEC-function highecut (see text). Uncertainties are at the 1 sigma (68\%) level. The maximum flux of the respective 35-day cycle is given in units of (ASM-cts s ${ }^{-1}$ ), referring to the All Sky Monitor of RXTE. The corresponding physical flux in units of $\left(\mathrm{keV} \mathrm{cm}^{-2} \mathrm{~s}\right)$ is found by multiplying with 0.2367 . The flux was measured by Swift/BAT and converted according to (2$10 \mathrm{keV})\left(\mathrm{ASM}-\mathrm{cts} \mathrm{s}^{-1}\right)=93.0 \times(15-50 \mathrm{keV})\left(\right.$ BAT-cts cm $\left.\mathrm{cm}^{-2} \mathrm{~s}\right)$ (Staubert et al. 2016). The observed line energy was normalized to an ASM count rate of $6.8 \mathrm{cts} \mathrm{s}^{-1}$ using a slope of $(0.675 \pm 0.075) \mathrm{keV} /\left(\mathrm{ASM}\right.$-cts s$\left.{ }^{-1}\right)$ (see Fig. 1$) .{ }^{(a)}$ We note that strength $=\sigma \tau \sqrt{2 \pi}$; ${ }^{(b)}$ the values for the CRSF are from Fürst et al. (2013), Obs. II (Table 3, HighE); ${ }^{(c)}$ in Staubert et al. $(2016,2017)$ the flux normalization was done with a slope of 0.44 (instead of 0.675); ${ }^{(d)}$ for the June 2019 observation only data from detector B have been used (see text); ${ }^{(e)} 35$-day cycle numbering is according to Staubert et al. (1983); ${ }^{(f)}$ observation at a high 35-day phase, $\Gamma$ expected to be lower (Vasco 2012).

Some of the listed NuSTAR observations were performed in coordination with other satellites, such as INTEGRAL, InsightHXMT, and Astrosat ${ }^{5}$, to study the CRSF. The observations in February and March 2019 were coordinated with XMM-Newton ${ }^{6}$ for a different project ${ }^{7}$, but also gave data on the CRSF. Here we do not report the results from the other satellites because we are still working on trying to resolve some inconsistencies, which are likely due to imperfect intercalibration between the different instruments and possibly aging of some of them ${ }^{8}$. We plan on reporting about this in a forthcoming paper. For first results from Insight-HXMT and Astrosat, see Xiao et al. (2019) and Bala et al. (2020).

\section{Results}

In Table 2 we summarize the results of the spectral analysis for the cyclotron line (the centroid energy, the width, and the strength) and for the continuum (cutoff energy $E_{\text {cut }}$, e-folding energy $E_{\text {fold }}$, and power-law index $\Gamma$ ). We further list the maximum fluxes of the respective 35-day cycles.

In order to allow us to compare our results with previous results, we used the observational flux units of (ASMcts s$^{-1}$ ), referring to the All Sky Monitor of RXTE) ${ }^{9}$, using the conversion $(2-10 \mathrm{keV})\left(\mathrm{ASM}-\mathrm{cts} \mathrm{s}^{-1}\right)=93.0 \times(15-50 \mathrm{keV})$ (BAT-cts $\mathrm{cm}^{-2} \mathrm{~s}$ ). This was found by Staubert et al. (2016) by comparing flux values measured by the All Sky Monitor on board RXTE on the one hand and those from Swift/BAT on the other, for the overlapping time of both missions.

INTEGRAL is the International Gamma-ray Astrophysics Laboratory of ESA, Insight-HXMT the Chinese mission Hard X-ray Modulation Telescope, and Astrosat the X-ray satellite mission of India.

6 XMM-Newton is ESAs Multi Mirror soft X-ray mission.

7 Brumback et al. (2020).

8 We are still attempting to perform further simultaneous observations with NUSTAR, INTEGRAL, and Insight-HXMT; some are already planned.

9 RXTE refers to the NASA mission Rossi X-ray Timing Explorer, and ASM to the All Sky Monitor on this satellite.
The relation between the ASM (or BAT) count rates and NuSTAR and corresponding physical flux units was established in the following way: The observed maximum ASM count rate (from the monitoring observations by Swift/BAT) for each $35 \mathrm{~d}$ cycle (Table 2) was plotted against the normalization, the flux at $1 \mathrm{keV}$, as determined through the spectral analysis of the corresponding NUSTAR observation (cycle 494 from February 2019 was excluded because this observation was at a 35 d-phase of 0.202 , after the maximum flux). This established the relation of a flux at $1 \mathrm{keV}$ [photons $\mathrm{cm}^{-2} \mathrm{~s} \mathrm{keV]}=0.0255 \times$ $\left(\mathrm{ASM}-\mathrm{cts} \mathrm{s}^{-1}\right)$, or a flux at $1 \mathrm{keV}$ [photons $\mathrm{cm}^{-2} \mathrm{~s} \mathrm{keV]}=$ $3.371 \times\left(\right.$ BAT-cts $\left.\mathrm{cm}^{-2} \mathrm{~s}\right)$. The energy flux was found by integrating the spectrum over the interested energy range. The following relationships in physical units emerge (taking the mean power-law index of -0.953$)$ : $(2-10 \mathrm{keV}$ flux)

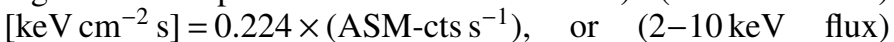

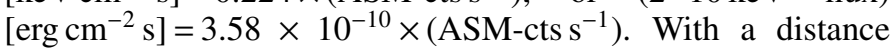
of $6.6 \mathrm{kpc}$ to Her X-1 (Reynolds et al. 1997), the corresponding $(2-10 \mathrm{keV})$ luminosities are given by $L_{X}(2-10 \mathrm{keV})$

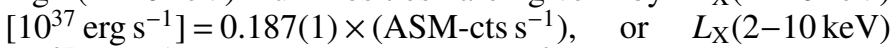
$\left[10^{37} \mathrm{erg} \mathrm{s}^{-1}\right]=17.4(1) \times\left(\right.$ BAT-cts cm $\left.{ }^{-2} \mathrm{~s}\right)$. We also list in Table 2 the cyclotron line energies normalized to a flux of 6.8 (ASM-cts s ${ }^{-1}$ ) using the determined linear flux dependence (Fig. 1) $E_{\text {cyc-norm }}[\mathrm{keV}]=E_{\text {cyc-obs }}[\mathrm{keV}]+0.675 \times(($ ASM$\left.\mathrm{cts} \mathrm{s}^{-1}\right)-6.8$ ). This relation is extremely well established (with a Pearson linear correlation coefficient of $0.98^{10}$ ). The choice of $6.8\left(\mathrm{ASM}\right.$-cts s${ }^{-1}$ ) as reference flux is historical and allows a direct comparison to previous results (for all other spectral parameters, we now use a reference flux of $5.0\left(\mathrm{ASM}_{\mathrm{C}} \mathrm{cts} \mathrm{s}^{-1}\right.$ ) because this flux is closer to the center of the flux range observed, and almost corresponds to a $(2-10 \mathrm{keV})$ luminosity of $\sim 10^{37} \mathrm{erg} \mathrm{s}^{-1}$ ).

Generally, all parameter values we state are derived from the combined spectral analysis of the two focal plane detectors of NUSTAR, except when stated otherwise.

${ }^{10}$ See, e.g., Numerical Recipes, W.H. Press et al., Cambridge University Press, 1986. 


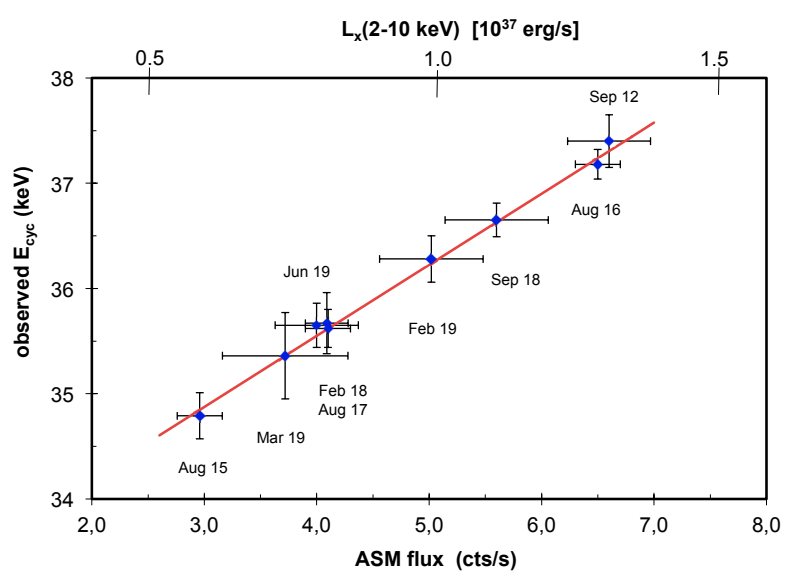

Fig. 1. Correlation between the measured values of the pulse-phaseaveraged cyclotron line energy and the X-ray flux (at the maximum of the respective $35 \mathrm{~d}$ main-on), as measured by Swift/BAT (in units of (ASM-cts s ${ }^{-1}$ )) for all NUSTAR observations between 2012 and March 2019 (see Table 1). We note that 1 (ASM$\mathrm{cts} \mathrm{s}^{-1}$ ) equals 93.0 (Swift/BAT-cts)/( $\mathrm{cm}^{2} \mathrm{~s}$ ) (Staubert et al. 2016) and $0.224\left(\mathrm{keV} \mathrm{cm}^{-2} \mathrm{~s}\right)$ in $(2-10 \mathrm{keV})$. The best-fit line defines a slope of $(0.675 \pm 0.075) \mathrm{keV} /\left(\mathrm{ASM}-\mathrm{cts} \mathrm{s}^{-1}\right)$. The Pearson linear correlation coefficient is 0.98 . For the mean power-law index of -0.953 and an adopted distance of $6.6 \mathrm{kpc}$ (Reynolds et al. 1997), the Her X-1 luminosity is $L_{\mathrm{X}}(2-10 \mathrm{keV})\left[10^{37} \mathrm{erg} \mathrm{s}^{-1}\right]=0.187(1) \times\left(\mathrm{ASM}^{-} \mathrm{cts} \mathrm{s}^{-1}\right)$.

\subsection{Cyclotron line parameters}

The new data allow us to determine the correlation between the observed pulse-averaged cyclotron line energy and the Xray flux with a significantly improved accuracy when compared to the time of the discovery of this correlation (Staubert et al. 2007). Figure 1 shows the definite correlation, which can be described by the linear function $E_{\text {cyc }}[\mathrm{keV}]=a+b \times$ ((ASM-cts) $\left.s^{-1}-6.8\right)$, with $a=(37.44 \pm 0.07)$, where the CRSF value is at an ASM count rate of $6.8 \mathrm{cts} \mathrm{s}^{-1}$ and $b=$ $(0.675 \pm 0.075) \mathrm{keV} /(\mathrm{ASM}$-cts $) \mathrm{s}^{-1}$ is the slope describing the flux dependence.

Figure 2 displays the evolution of the normalized CRSF centroid energy of Her X-1 from 2009 to 2019. The red data points are historical results that were published before, and the dashed line represents the end of the phase of the long-term decay of $E_{\text {cyc }}$ between 1996 and 2012, as reported by Staubert et al. (2016) (their Fig. 2). The right-hand side shows the latest values from NuSTAR (2015-2019). All data points are flux corrected (normalized to an ASM count rate of $6.8 \mathrm{cts} \mathrm{s}^{-1}$ ). The values since 2015 are apparently consistent with a constant value; the formal weighted average is $\left\langle E_{\text {cyc }}\right\rangle(2015-2019)=(37.44 \pm$ $0.07) \mathrm{keV}$. Because there is no time dependence of the normalized $E_{\text {cyc }}$ in 2015-2019, it is not necessary to perform a combined fit with simultaneously existing flux and time dependencies, as was done for the data before 2012 (Staubert et al. 2016).

We need to point out, however, that the new data require a modification of the view presented in Staubert et al. (2017), in which it was suggested that an inversion (an upward trend) in $E_{\text {cyc }}$ had occurred after the end of the decay. This impression was mainly driven by the 2015 measurement, which was made at an extremely low flux level, even the lowest of all NuSTAR observations at around 3 (ASM-cts) $\mathrm{s}^{-1}$ (see Fig. 1). On the one hand, this was useful in extending the dynamic range in observed fluxes beyond the (classical) factor of two, but on the other hand, it led to a very low value of the flux normalized $E_{\text {cyc }}$ when the correction was made with the best value of the flux dependence of $0.44 \mathrm{keV} /\left(\mathrm{ASM}-\mathrm{cts} \mathrm{s}^{-1}\right.$ ) (Staubert et al. 2016, 2017). When the current best value $(0.675$ instead of 0.44$)$ is used for the normalization, the 2015 value is significantly higher and consistent with values found throughout 2012-2019 (see Table 1 and Fig. 2).

A further result of our analysis of the nine NuSTAR observations is that all other characteristic parameters of the cyclotron line, that is, the width $\sigma$, the strength, the optical depth $\tau$, and the relative width, are also linearly correlated with the X-ray flux. In Table 3 we summarize the results of linear fits of all the CRSF line parameters versus flux. This also means that all line parameters linearly correlate with one another. As an example, Fig. 3 shows the dependence of the line width $\sigma$ on flux and Fig. 4 the dependence of $\sigma$ on $E_{\text {cyc. This correlation is }}$ a well-known behavior (apparently valid for all cyclotron line sources, e.g., Makishima et al. 1999; Coburn et al. 2002) that is expected to occur through thermal Doppler broadening because electrons move freely along the magnetic field lines (see discussion below). Even though the correlations between the different parameters can in principle be reconstructed from the respective dependencies of all parameters on flux (Table 3), we performed the linear fits for every possible pair of parameters explicitly and summarize the results in Table 4.

We note that the relative line width, $\sigma / E_{\text {cyc }}$, is not constant, but increases with increasing flux (Fig. 5) according to $\sigma / E_{\text {cyc }}=0.146+0.0087(\mathrm{ASM}-5.0)$. This means that the relative change with changing flux is stronger for the line width than for the line energy.

In addition, we give the linear correlation between the relative line width $\sigma / E_{\text {cyc }}$ to the optical depth $\tau$ (Fig. 6), a correlation first noted by Coburn et al. (2002) in a group of cyclotron line objects. As with other correlations, this one can also be realized in individual objects, here Her X-1, but also in 4U 1538-52 (Rodes-Roca et al. 2008). This may not be so easy to understand in the context of theoretical considerations (see discussion). In Fig. 7 the value of $E_{\text {cut }}$ from observation in June 2019 (cycle 498) was taken from focal plane detector B only. The value from detector A is exceptionally high and far outside the overall trend (the anomaly is being investigated).

\subsection{Continuum parameters}

The systematic monitoring of Her X-1 with $N u S T A R$ over the past decade has allowed the discovery that all continuum parameters, $E_{\text {cut }}, E_{\text {fold }}$, and $\Gamma$ are systematically correlated with X-ray flux. The correlations can be described by linear functions, see Table 3, Figs. 7, 8 and 9. Because both $E_{\text {cut }}$ and $E_{\text {fold }}$ depend on X-ray flux, they correlate with each other, which is shown in Fig. 10. Normalizing $E_{\text {cut }}$ and $E_{\text {fold }}$ to the reference flux of 5.0 (ASM-cts s ${ }^{-1}$ ), we find that both normalized parameters (excluding the values of cycle 494 in February 2019, where the 35dphase is very high, see Sect. 3.4) are consistent with a constant value over the time span 2012-2019 (Fig. 11). In calculating the dependence of $\Gamma$ on flux, we excluded the exceptionally low value (0.885) measured in February 2019 (cycle 494, see Table 2) because the observation was made at a high 35-day phase of 0.202 , where the flux is about $65 \%$ of the maximum main-on flux of this cycle. At 35 -day phases beyond $\sim 0.16, \Gamma$ is known to decrease (Vasco 2012). The measured increase of $\Gamma$ on flux is fairly weak but interesting because this disagrees with reports about a correlation in the opposite sense by Klochkov et al. (2011) (see the discussion below). 


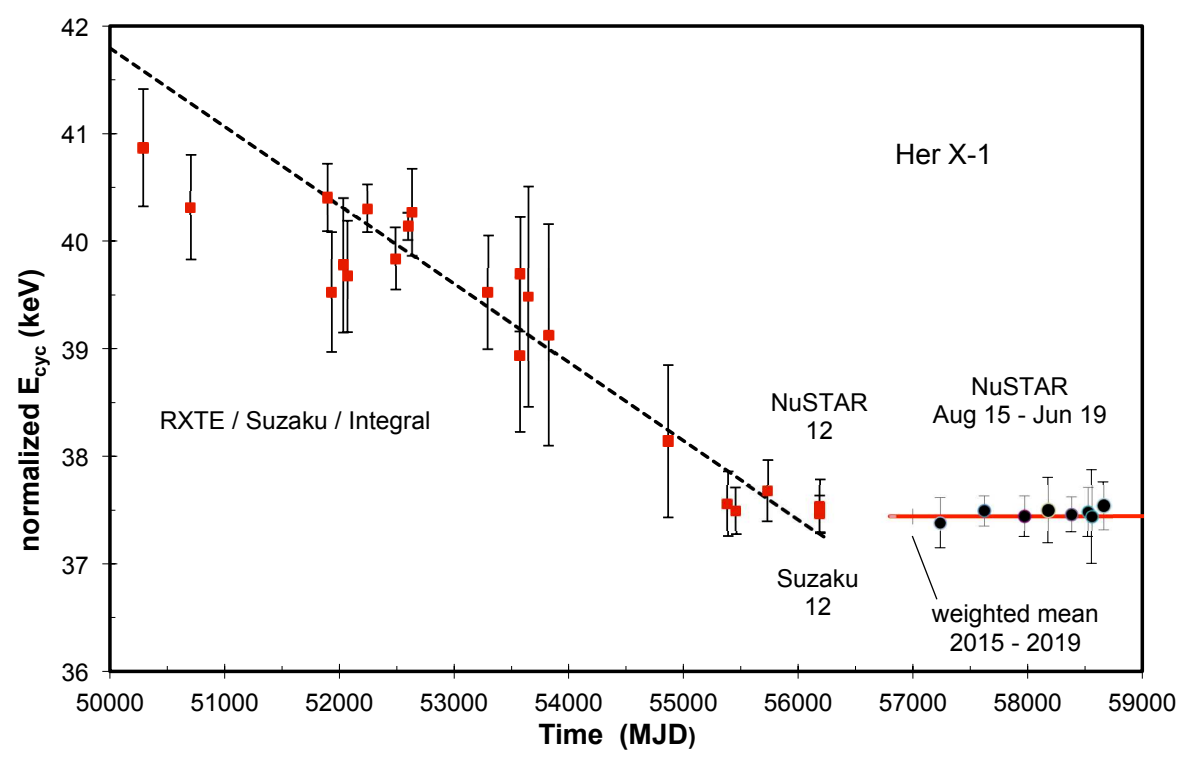

Fig. 2. Evolution of the cyclotron line energy $E_{\text {cyc }}$ in Her X-1. The red points until 2012 (MJD 56200) and the corresponding linear best fit (dashed line) are reproduced from Staubert et al. (2016). The black points are the new measurements by NUSTAR from 2015-2019 (see Table 1): the pulse-phase-averaged $E_{\text {cyc }}$ values normalized to a reference ASM count rate of 6.8 (ASM-cts s ${ }^{-1}$ ) using a flux dependence of $0.675 \mathrm{keV} /\left(\right.$ ASM-cts s$^{-1}$ ) (see Fig. 1). The solid red line represents the weighted mean of $(37.44 \pm$ $0.07) \mathrm{keV}$, demonstrating a constant value since at least 2012.
Table 3. Linear dependence of spectral parameters on X-ray flux.

\begin{tabular}{llll}
\hline \hline Parameters & $\begin{array}{l}a[\mathrm{keV}] \text { or } \\
\text { no dimension }\end{array}$ & $b^{(d)}$ & $\begin{array}{l}\text { Pearson } \\
\text { corr. coeff. }\end{array}$ \\
\hline$E_{\text {cyc }}{ }^{(a)}[\mathrm{keV}]$ & $36.24 \pm 0.09$ & $0.675 \pm 0.075$ & 0.98 \\
$\sigma_{\text {cyc }}{ }^{(b)}[\mathrm{keV}]$ & $5.30 \pm 0.09$ & $0.41 \pm 0.07$ & 0.99 \\
Strength $^{(c)}$ & $7.28 \pm 0.22$ & $1.15 \pm 0.19$ & 0.96 \\
${\text { Opt. depth } \tau^{(c)}}^{(0.54 \pm 0.02}$ & $0.042 \pm 0.015$ & 0.87 \\
$\sigma_{\text {cyc }} / E_{\text {cyc }}$ & $0.146 \pm 0.002$ & $0.0086 \pm 0.0002$ & 0.97 \\
$E_{\text {cut }}[\mathrm{keV}]$ & $20.17 \pm 0.06$ & $0.30 \pm 0.06$ & 0.74 \\
$E_{\text {fold }}[\mathrm{keV}]$ & $9.83 \pm 0.04$ & $0.24 \pm 0.03$ & 0.79 \\
$\Gamma$ & $0.965 \pm 0.002$ & $0.015 \pm 0.001$ & 0.53 \\
\hline
\end{tabular}

Notes. The five line parameters are the centroid energy $E_{\text {cyc }}$, the width $\sigma_{\text {cyc }}$, the line strength, the optical depth $\tau$ (see Eq. (2)), and the relative width $\sigma_{\text {cyc }} / E_{\text {cyc }}$. The three continuum parameters are $E_{\text {cut }}$ and $E_{\text {fold }}$ and the power-law photon index $\Gamma$. The $\mathrm{X}$-ray flux is measured in units of (ASM-cts s ${ }^{-1}$ ), referring to the All Sky Monitor of (RXTE): $y=a+b(x-$ c). Here, the offset in $\mathrm{x}$ is always constant: $c=5.0$ (ASM-cts ${ }^{-1}$ ) (close to the center of the range of fluxes observed). We note that 5.0 (ASMcts s$\left.^{-1}\right)$ corresponds to $5.0 \times 0.237=1.18\left(\mathrm{keV} \mathrm{cm}^{-2} \mathrm{~s}\right)$ in $(2-10 \mathrm{keV})$. Uncertainties are at the 1 sigma (68\%) level. The last column lists the Pearson linear correlation coefficients. ${ }^{(a)}$ Figure $1 ;{ }^{(b)}$ Fig. $3 ;{ }^{(c)}$ we note

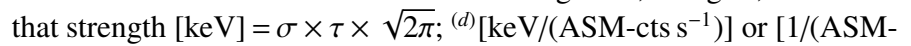
cts s $\left.\left.^{-1}\right)\right]$.

\subsection{Correlation between line and continuum parameters}

Because the continuum parameters $E_{\text {cut }}$ and $E_{\text {fold }}$ and all cyclotron line parameters correlate with X-ray flux, the line and the continuum parameters are also correlated. As two examples we list the linear correlation parameters of the observed cyclotron line energy and the continuum parameters in Table 4 and show them in Figs. 12 and 13.

\subsection{Dependence on phase of the 35-day modulation}

As mentioned in the Introduction, Her X-1 shows a regular 35-day modulation that has been known since the discovery of the source by UHURU (Tananbaum et al. 1972) and is thought to be connected with the precession of the accretion disk that provides regular shadowing of the $\mathrm{X}$-ray source. The 35 -day peri- odicity is also seen in the regular variations in the shape of the pulse profiles (Trümper et al. 1986; Staubert et al. 2013), which has also led to the suggestion that free precession of the neutron star may play a role (Trümper et al. 1986; Postnov et al. 2013); this is still an open question and highly debated. In the context of this work, it is of interest whether the X-ray spectra show variations with phase of the 35-day modulation. This is indeed the case (Parmar et al. 1980; Mihara et al. 1991; Kuster et al. 2005; Vasco 2012). Staubert et al. (2014) suggested that there is a weak modulation of the cyclotron line energy $E_{\text {cyc }}$ during the main-on, and Vasco (2012) reported a strong variation in the power-law index for 35-day phases greater than 0.16 . The contribution of the series of NUSTAR observations of Her X-1, discussed here, is the following (for the limited range provided by the data used here, see Table 1): (1) The flux-normalized $E_{\text {cyc }}$ does not show any variation, the values are consistent with a constant. (2) The flux-normalized values of $E_{\text {cut }}$ and $E_{\text {fold }}$ are constant up to the second highest 35 -day phase observed $(0.147$ for Aug 2017, cycle 478), then both drop to significantly lower values for the highest observed 35 -day phase ( 0.202 for February 2019, cycle 494). (3) The normalized power-law index $\Gamma$ behaves as the other two continuum parameters and drops to the lowest value (0.885) for the February 2019 observation at 35-day phase 0.202 .

\section{Discussion}

Correlations between spectral parameters were first discovered by comparing spectral parameters determined for different objects (Makishima \& Mihara 1992; Makishima et al. 1999; Coburn et al. 2002). We now see, most prominently (but not only) in Her X-1, that strong correlations between spectral parameters, both continuum and line parameters, exist for individual objects, particularly related to changes in X-ray luminosity.

\subsection{Correlation between line parameters}

The linear correlation between the width of the cyclotron line and its centroid energy (Fig. 4) is expected because the line is Doppler-broadened as a result of the thermal motion of the electron gas at a temperature $k T_{\mathrm{e}}$. Applying the general 


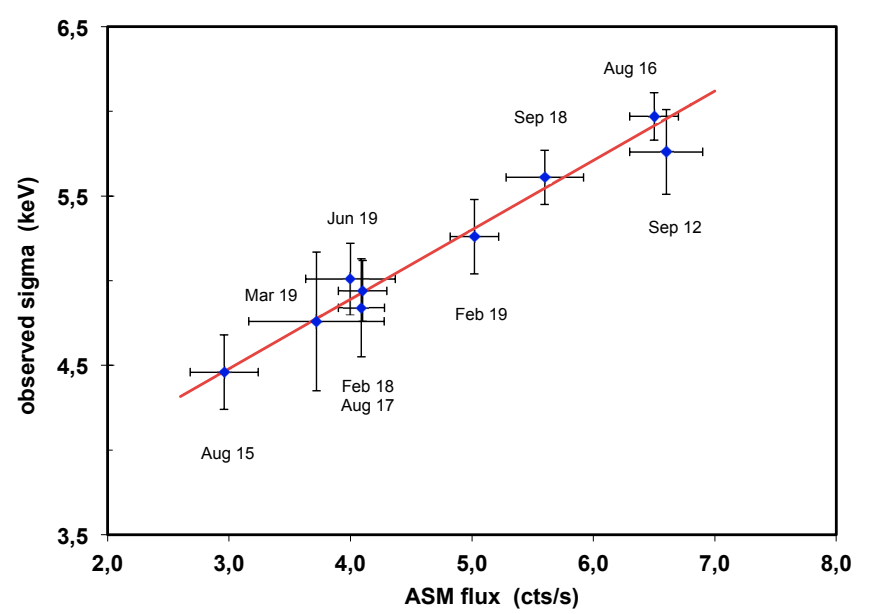

Fig. 3. Correlation between the measured values of the width (sigma) of the cyclotron line and the X-ray flux. The fit takes into account nine measurements by NuSTAR from 2012 to 2019 (see Table 1). The best-fit line is given by the function $\operatorname{sig}_{\mathrm{cyc}}=(5.30 \pm 0.09)+$

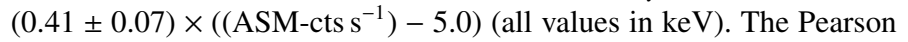
linear correlation coefficient is 0.99 .

formula for a Doppler-broadened line (of central energy $E$ ) to the resonant cyclotron scattering of photons on electrons, we write $\sigma=E\left(k T_{\mathrm{e}} / m_{\mathrm{e}} c^{2}\right)^{1 / 211}$. Because electrons in a strong magnetic field can move freely only in one dimension (along the field lines), we need to multiply by $\cos \theta$, where $\theta$ is the angle between the viewing direction and the magnetic field lines. The temperature of the electron gas is $k T_{\mathrm{e}}$, and the rest mass of the electron is $m_{\mathrm{e}} c^{2} \approx 511 \mathrm{keV}$, so that the electron temperature can be estimated to be $k T_{\mathrm{e}}[\mathrm{keV}] \approx 511\left(\sigma / E_{\text {cyc }}\right)^{2} /\left|\cos ^{2} \Theta\right|$. The line-broadening effect has been pointed out by Trümper et al. (1977) when the discovery of the first cyclotron line, in Her X-1, was reported (see also Meszaros \& Nagel 1985; Orlandini et al. 1998). It was then observationally confirmed when different cyclotron line energies and associated widths in several X-ray binaries were measured (Makishima et al. 1999; Coburn et al. 2002). Recently, it became possible to observe such correlations in individual sources, when the CRSF energy as well as the line width change with varying flux. Here we report the measurement for Her X-1: $\sigma=5.26+0.60\left(E_{\mathrm{cyc}}-36.0\right)$ (all in $\left.\mathrm{keV}\right)$. For small $\theta(\cos \theta \sim 1)$, the calculated $k T_{\mathrm{e}}$ ranges from $8.3 \mathrm{keV}$ to $13.5 \mathrm{keV}$ for flux values from 3 to $7 \mathrm{ASM} \mathrm{Ats} \mathrm{s}^{-1}$, respectively. This is very close to $10 \mathrm{keV}$, which is the typical value of the continuum parameter $E_{\text {fold }}$, often taken as the temperature of the plasma that emits the continuum. It is tempting to conclude that in Her X-1 we most likely see a pencil beam rather than a fan beam.

The fact that the relative line width $\sigma / E_{\text {cyc }}$ increases with increasing flux (Fig. 5) means that the electron temperature $k T_{\mathrm{e}}$ increases with increasing flux, and so does $E_{\text {fold }}$, as expected for an increasing accretion rate. However, the magnitude of the increase in $k T_{\mathrm{e}}$ is $\sim 12 \%$ per unit flux, significantly stronger than the increase in $E_{\text {fold }}$ with only $\sim 2 \%$. If $E_{\text {fold }}$ is indeed a measure of the continuum temperature, then the electron temperature is increasing significantly faster.

The general dependence of $\sigma$ on $E_{\text {cyc }}$ for the known cyclotron line objects is demonstrated in Fig. 14 (up to $E_{\text {cyc }}=60 \mathrm{keV}$ ), where pairs of $\sigma$ and $E_{\text {cyc }}$ values are shown,

11 The general formula for a Doppler-broadened line is $F W H M=$ $E \times\left(8 \ln 2 k T_{\mathrm{e}} / m_{\mathrm{e}} c^{2}\right)^{1 / 2}$, where $E$ is the line energy and $F W H M=$ $\sigma(8 \ln 2)^{1 / 2}=2.356 \sigma$ is the full width at half maximum (see, e.g., K.R. Lang, Astrophysical Formulae, Springer).

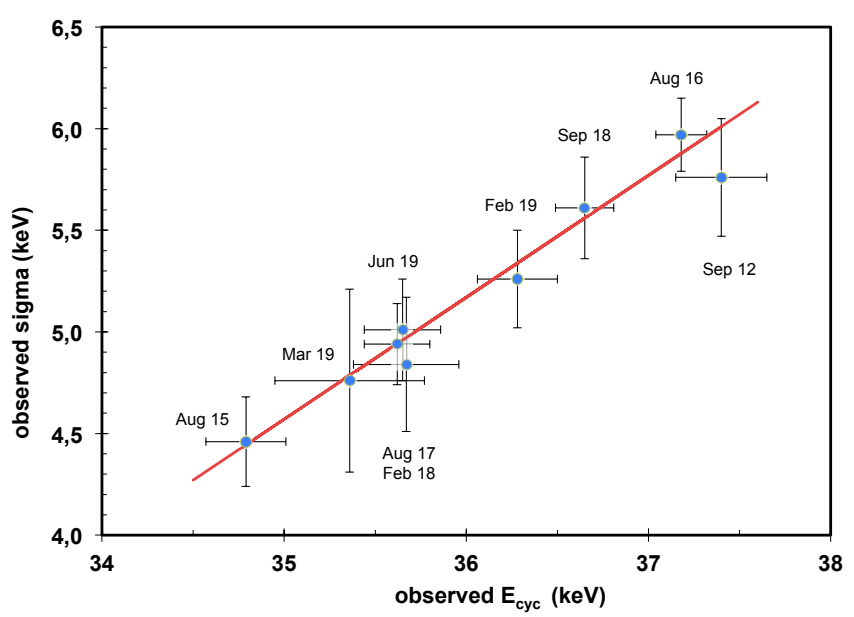

Fig. 4. Correlation between the width (sigma) of the pulse-phaseaveraged cyclotron line and its centroid energy. The fit takes into account nine measurements by NUSTAR from 2012 to 2019 (see Table 1). The best-fit line is given by the function $\sigma_{\mathrm{cyc}}=(5.17 \pm 0.09)+$ $(0.60 \pm 0.11) \times\left(E_{\text {cyc }}-36.0\right)$ (all values in $\left.\mathrm{keV}\right)$. The Pearson linear correlation coefficient is 0.96 .

taken from Coburn et al. (2002) and Staubert et al. (2019) (their Table A.5), together with a few individual objects. The central line through the rather scattered data defines a slope of $\sim 0.18 \mathrm{keV} \mathrm{keV}^{-1}$, corresponding to a mean $k T_{\mathrm{e}}$ of $\sim 16 \mathrm{keV}$ for $\Theta=0$. For a few objects we have now observed variations of both the line energy and the line width (physically introduced by a changing X-ray flux): Her X-1 (Staubert et al. 2007 and this work), GX 304-1 (Klochkov et al. 2015; Malacaria et al. 2015; Rothschild et al. 2017), Vela X-1 (La Parola et al. 2016) (the first harmonic), and Swift J1626.6-5156 (DeCesar et al. 2013) (see Fig. 14). For these few objects, the absolute values of the relative widths are all rather low (they are all below the red line in Fig. 14, but the variation $\mathrm{d}(\sigma) / \mathrm{d}\left(E_{\text {cyc }}\right)$ is significantly steeper than for the complete ensemble; e.g., for Her X-1, it is $0.60 \mathrm{keV} \mathrm{keV}^{-1}$ ).

We would like to stress that the X-ray flux (physically the mass accretion rate) is a fundamental parameter that seems to affect all spectral parameters. In addition to the line position and width, its strength, its depth, and its relative width are positively correlated with flux (see Table 3). The same is true for the continuum parameters $E_{\text {cut }}$ and $E_{\text {fold }}$ (Figs. 7 and 8), and surprisingly (even if weak), for the power-law index $\Gamma$. We find that $\Gamma$ increases with flux (by $1.8 \%$ for an increase in flux by a factor of two), while Klochkov et al. (2011), in pulse-amplituderesolved spectroscopy of Her X-1, found the opposite trend (by $5.6 \%$ ), always in combination with an increase in $E_{\text {cyc }}$ with flux. A solution may be given by Postnov et al. (2015), who showed (for several sources, but unfortunately not for Her X-1) that the spectral hardness correlates with X-ray flux, consistent with Klochkov et al. (2011), but only up to a (source-dependent) luminosity of about a few times $10^{37} \mathrm{erg} \mathrm{s}^{-1}$, after which the correlation stops or even reverses. A luminosity of a few times $10^{37} \mathrm{erg} \mathrm{s}^{-1}$ is considered to be close to the border between the sub- and supercritical accretion regimes (Becker et al. 2012) at which the trend for these correlations reverses. Her X-1 probably operates close to the critical luminosity, and the turning point for a reversal may even be slightly different between the respective correlations.

An interesting correlation, first found by Coburn et al. (2002) in a group of X-ray binaries, namely the relative line width 
Table 4. Relations between line parameters, $y=a+b(x-c)$ : parameter $y$ (top line) vs. parameter $x$ (first column).

\begin{tabular}{|c|c|c|c|c|c|}
\hline $\begin{array}{l}\text { Parameter } \\
\text { versus }\end{array}$ & $\begin{array}{l}E_{\text {cyc }} \\
{[\mathrm{keV}]}\end{array}$ & $\begin{array}{l}\sigma_{\text {cyc }} \\
{[\mathrm{keV}]}\end{array}$ & $\begin{array}{l}\text { Strength } \\
{[\mathrm{keV}]}\end{array}$ & $\tau$ & $\sigma_{\mathrm{cyc}} / E_{\mathrm{cyc}}$ \\
\hline$E_{\mathrm{cyc}}$ & $\begin{array}{l}- \\
- \\
-\end{array}$ & $\begin{array}{l}a=5.17 \pm 0.09 \\
b=0.60 \pm 0.11 \\
c=36.0 \mathrm{keV}\end{array}$ & $\begin{array}{l}a=6.91 \pm 0.22 \\
b=1.71 \pm 0.29 \\
c=36.0 \mathrm{keV}\end{array}$ & $\begin{array}{l}a=0.53 \pm 0.02 \\
b=0.064 \pm 0.023 \\
c=36.0 \mathrm{keV}\end{array}$ & \\
\hline$\sigma_{\text {cyc }}$ & & $\begin{array}{l}- \\
- \\
- \\
\end{array}$ & $\begin{array}{l}a=6.42 \pm 0.31 \\
b=2.84 \pm 0.63 \\
c=5.0 \mathrm{keV}\end{array}$ & $\begin{array}{l}a=0.51 \pm 0.02 \\
b=0.11 \pm 0.04 \\
c=5.0 \mathrm{keV}\end{array}$ & \\
\hline Strength & & & $\begin{array}{l}- \\
- \\
-\end{array}$ & $\begin{array}{l}a=0.53 \pm 0.02 \\
b=0.041 \pm 0.014 \\
c=7.0\end{array}$ & \\
\hline$\tau$ & & & & $\begin{array}{l}- \\
- \\
-\end{array}$ & $\begin{array}{l}a=0.14 \pm 0.05 \\
b=0.19 \pm 0.08 \\
c=0.50\end{array}$ \\
\hline$E_{\text {cut }}$ & $\begin{array}{l}a=35.86 \pm 0.11 \\
b=1.87 \pm 0.36 \\
c=20.0 \mathrm{keV}\end{array}$ & & & & \\
\hline$E_{\text {fold }}$ & $\begin{array}{l}a=36.69 \pm 0.13 \\
b=2.71 \pm 0.39 \\
c=10.0 \mathrm{keV}\end{array}$ & & & & \\
\hline
\end{tabular}

Notes. The offset $c$ is constant for any given parameter $x$. Uncertainties are at the 1 sigma (68\%) level.

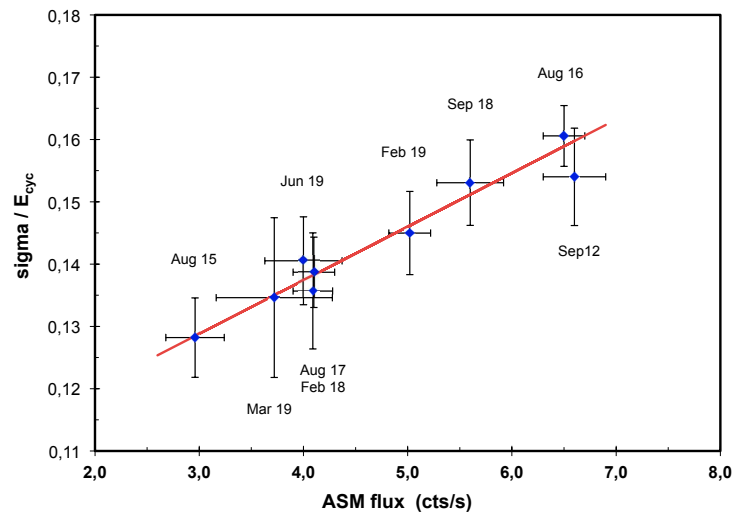

Fig. 5. Relative width of the cyclotron line vs. X-ray flux in units of $\left(\mathrm{ASM}-\mathrm{cts} \mathrm{s}^{-1}\right)$. The best-fit line is given by the function $\sigma_{\text {cyc }} / E_{\text {cyc }}=(0.146 \pm 0.002)+(0.0086 \pm 0.0019) \times\left(\left(\right.\right.$ ASM-cts s $\left.\left.^{-1}\right)-5.0\right)$. The Pearson linear correlation coefficient is 0.97 .

$\sigma_{\text {cyc }} / E_{\text {cyc }}$ as function of the optical depth $\tau$, is also realized in individual objects such as 4U 1538-52 (Rodes-Roca et al. 2008) and in HerX-1 (Fig. 6). Coburn et al. (2002) and Rodes-Roca et al. (2008) noted that simple theoretical models of cyclotron line generation predict the opposite dependence (Isenberg et al. 1998; Araya \& Harding 1999).

Regarding intercorrelations between spectral parameters, it has been a general worry about how large the effect of purely mathematical correlations is that are introduced in the fitting process. Coburn et al. (2002) have tried to answer this question by performing Monte Carlo simulations, carefully designed for different types of correlations. Their conclusion was that formal correlations were not significant and that it was safe to conclude that the observed correlations are a true physical effect. We used the Markov chain Monte Carlo (MCMC) procedure offered in XSPEC to investigate the same question and present a corresponding analysis in Appendix A. We are confident that the observed correlations are indeed physical.

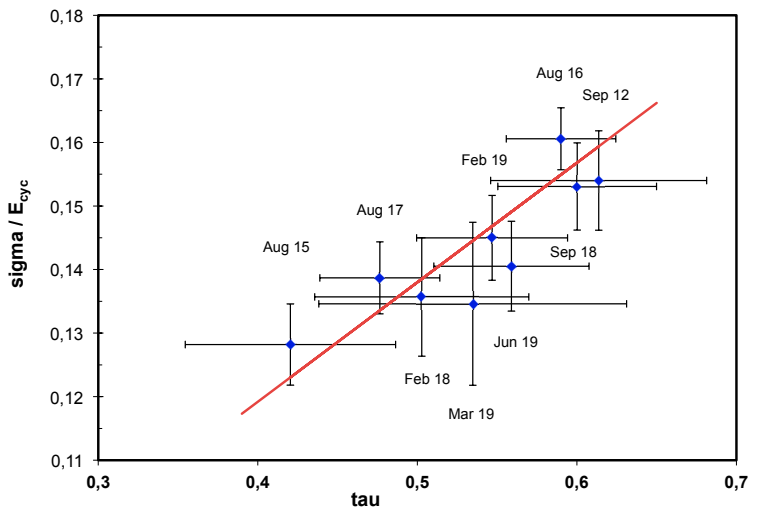

Fig. 6. Relative width of the cyclotron line vs. optical depth $\tau$. The best-fit line is given by the function $\sigma_{\text {cyc }} / E_{\text {cyc }}=(1.38 \pm 0.005)+$ $(0.188 \pm 0.076) \times\left(\tau_{\mathrm{cyc}}-0.5\right)$. The Pearson linear correlation coefficient is 0.88 .

\subsection{Physics behind changes in $E_{\mathrm{cyc}}$}

The centroid energy of the cyclotron line in Her X-1 has been observed to change with respect to four parameters (Staubert et al. 2014). The first is pulse phase: 20\% ((max$\mathrm{min}$ )/mean) (Voges et al. 1982; Vasco et al. 2013; Staubert et al. 2014); The second is X-ray flux: $6.5 \%$ for a change in flux by a factor of two (here and Staubert et al. 2007); The third is elapsed time: constant around $35 \mathrm{keV}$ from the discovery in 1975 to 1990 , jump upward 1991-1994 from $\sim 35 \mathrm{keV}$ to beyond $40 \mathrm{keV}(\sim 20 \%)$ (Gruber et al. 2001; Staubert et al. 2007), followed by a well-measured decay until $\sim 2012$ to $\sim 37 \mathrm{keV}$ (10\% over 16 years) (here and Staubert et al. 2014, 2016); the fourth is possibly the phase of the 35-day on-off cycle with a change by $1 \mathrm{keV}$ or less (Staubert et al. 2014), which we do not confirm here.

The variation with pulse phase is believed to be due to the changing viewing angle under which the emission regions 


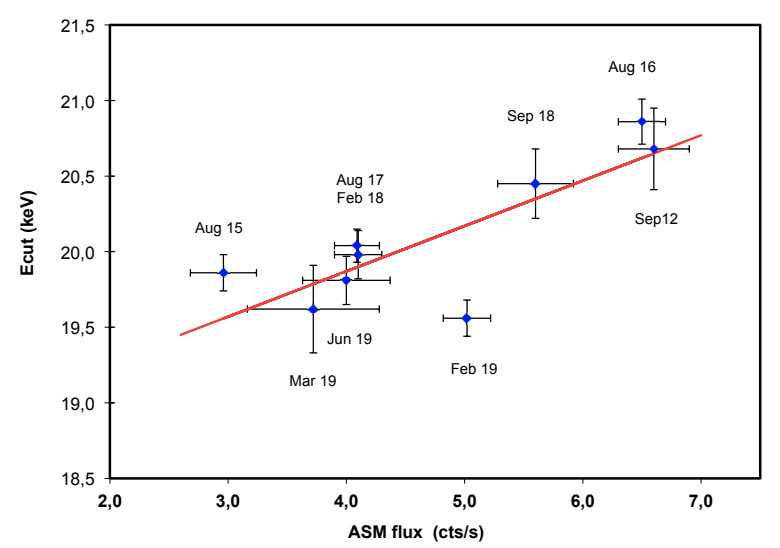

Fig. 7. Continuum parameter $E_{\text {cut }}$ vs. X-ray flux in units of (ASMcts s$^{-1}$ ). The value for the June 2019 observation is from focal plane detector B only (see text). The best-fit line is given by the function $E_{\text {cut }}=(20.17 \pm 0.06)+(0.30 \pm 0.06) \times(\mathrm{ASM}-5.0)($ all values in $\mathrm{keV})$. The Pearson linear correlation coefficient is 0.74

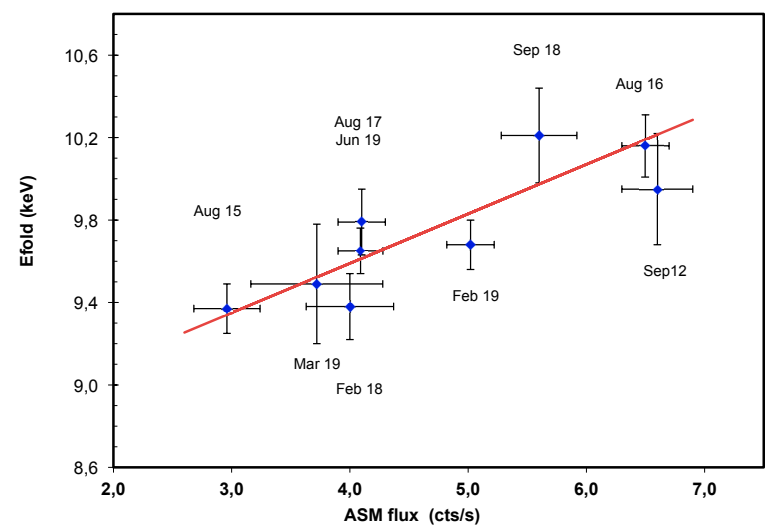

Fig. 8. Continuum parameter $E_{\text {fold }}$ vs. X-ray flux in units of (ASM$\left.\operatorname{cts~s}^{-1}\right)$. The best-fit line is given by the function $E_{\text {fold }}=(9.83 \pm 0.04)+$ $(0.24 \pm 0.03) \times\left(\left(\mathrm{ASM}^{-\mathrm{ct} \mathrm{s}^{-1}}\right)-5.0\right)($ all values in $\mathrm{keV})$. The Pearson linear correlation coefficient is 0.79 .

are seen (Schönherr et al. 2007). Vasco (2012) showed that the above discussed correlation between the width and the centroid energy of the cyclotron line (and the dependence of both on flux) is also valid when certain pulse phases are analyzed (e.g., the line energy and the width are both at a maximum around the peak of the main pulse). We do not discuss this phenomenon further here, but concentrate on the dependence of the pulse-phase-averaged cyclotron line energy $E_{\text {cyc }}$ on X-ray flux and on time.

\subsubsection{Changes in $E_{\text {cyc }}$ with flux}

With respect to the physics at work behind the positive correlation of the pulse-phase-averaged cyclotron line energy with flux, we refer to discussions presented earlier by Staubert et al. (2007, 2014, 2016, 2017) and Ji et al. (2019), as well as the theoretical work by Becker et al. (2012) (see also the summary and references given in the review by Staubert et al. 2019). Here detailed modeling of the physics is necessary, led by the question of defining the deceleration mechanism of the accreted material. Is it due to simple Coulomb scattering or the generation of radiative shocks? What type of accretion rate is necessary to generate such shocks, and at which height above the neutron star surface would they form? What is the configuration of the magnetic field, most likely influenced by the in-

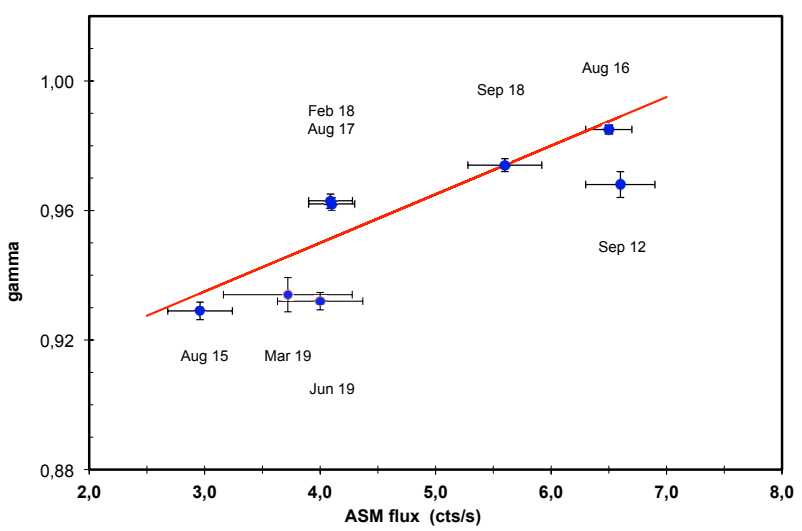

Fig. 9. Power-law index $\Gamma$ vs. flux in $\left(A S M-c t s ~^{-1}\right)$. The best-fit line is given by the function $\Gamma=(0.965 \pm 0.002)+(0.015 \pm 0.001) \times((\mathrm{ASM}-$ $\left.\left.\mathrm{cts} \mathrm{s}^{-1}\right)-5.0\right)$. This function is valid for 35 -day phases up to 0.16 . The low $\Gamma$ value measured in February 2019 (cycle 494, see Table 2) was not included in this fit because the observation took place at a 35-day phase of 0.202 , at which a lower value is expected (see text). The Pearson linear correlation coefficient is 0.53 .

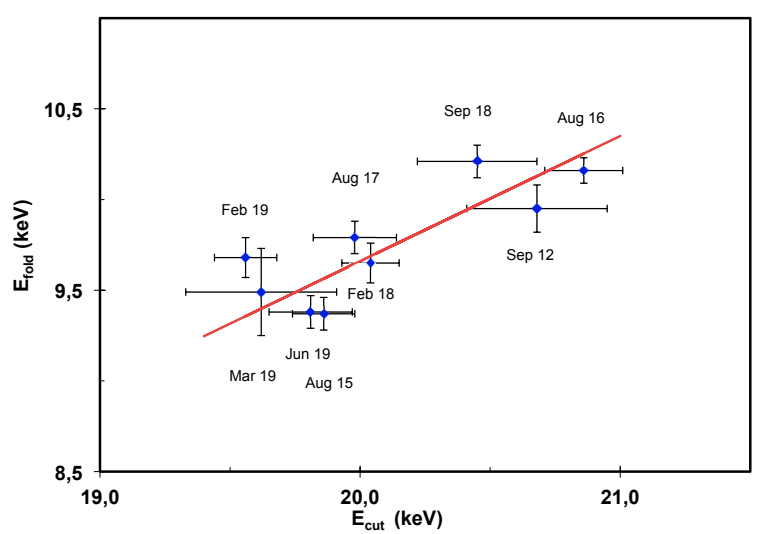

Fig. 10. Continuum parameter $E_{\text {fold }}$ vs. the continuum parameter $E_{\text {cut }}$. The best-fit line is given by the function $E_{\text {fold }}=(9.66 \pm 0.05)+(0.69 \pm$ $0.12) \times\left(E_{\text {cut }}-20.0\right)$ (all values in $\left.\mathrm{keV}\right)$. The Pearson linear correlation coefficient is 0.82 .

falling material? It has become popular to talk about accretion regimes (e.g., Becker et al. 2012), which can be subcritical and supercritical, most likely separated by a critical luminosity of about a few times $10^{37} \mathrm{erg} \mathrm{s}^{-1}$. Generally, subcritical accretion is associated with a positive $E_{\text {cyc }} / L_{\mathrm{X}}$ correlation, and supercritical accretion with a negative correlation (Becker et al. 2012; Mushtukov et al. 2015). Recently, a model involving a collisionless shock was developed that also explains the deviation from a pure linear dependence (a roll-off), as observed in GX 304-1 (Rothschild et al. 2017; Vybornov et al. 2017). A more detailed discussion is presented in Staubert et al. (2017).

Alternatively, or in combination with a height-related effect, the observed variations could be due to changes of the configuration of the magnetic field when the accretion rate varies. As Mukherjee et al. $(2013,2014)$ showed, the usually assumed dipole structure of the magnetic field is significantly altered when the mass accretion rate changes. Close to the magnetic poles, a higher accretion rate can lead to a significant increase in the density of field lines at the outer circumference of the accretion mound when the infalling material pushes matter and field lines from the center radially outward. See also the discussion in Bala et al. (2020). 


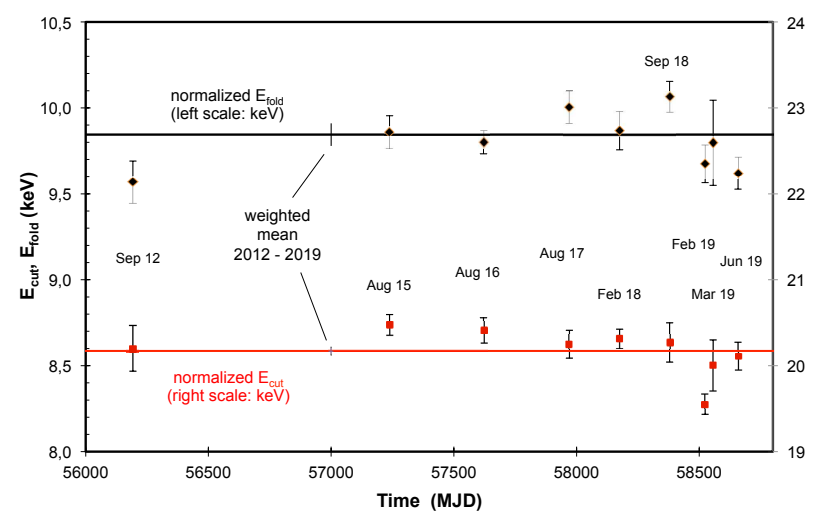

Fig. 11. Flux-normalized values of $E_{\text {cut }}$ (right scale) and $E_{\text {fold }}$ (left scale) as functions of time. The normalization to a reference X-ray flux of 5 (ASM-cts s ${ }^{-1}$ ) uses the linear correlations as stated in Table 3 and shown in Figs. 7 and 8. These two continuum parameters appear to be constant (see the horizontal lines) in 2012-2019, except for February 2019 , where the $35 \mathrm{~d}$-phase is very high (0.202).

\subsubsection{Changes in $E_{\text {cyc }}$ with time}

The dependence of $E_{\text {cyc }}$ on time is even less well understood. With regard to the long-term decay of $E_{\text {cyc }}$, it is probably either a geometric displacement of the emission region or a change in the local field configuration, as calculated by Mukherjee \& Bhattacharya (2012), for instance, rather than a change in the strength of the underlying global dipole. Staubert (2014) suggested that the observed change in $E_{\text {cyc }}$ may be connected to a slight imbalance between gain and loss of accreted material, such that the structure of the accretion mound changes with time. With an accretion rate of $\sim 10^{17} \mathrm{~g} \mathrm{~s}^{-1}$, a variation on relatively short timescales seems plausible. If the gain is slightly higher than the loss, material would slowly accumulate in the mound, possibly increasing its height or changing the local field structure, which might be the reason for the long-term reduction in $E_{\text {cyc }}$. This reduction might be expected to find a natural end (e.g., when the excess mass and the associated pressure in the accretion mound becomes too high), such that a forced outflow of material to larger areas of the NS surface causes a readjustment of the accretion mound back to the unperturbed configuration. This could be a relatively fast and catastrophic event, possibly explaining the rather sudden upward jump in $E_{\text {cyc }}$ observed between 1990 and 1993. The time period 1990-2012 sets an apparent timescale of instability: a few years of very fast change (the increase in $E_{\text {cyc }}$ by $\sim 5 \mathrm{keV}$ ), followed by $\sim 16$ years of decay to the original level. A corresponding timescale for stability is not yet known. Future observations should search for indications for an increase or even a new upward jump in $E_{\text {cyc }}$. Because an upward movement could be rather fast (similar to the earlier event), it is important to observe as regularly as possible so as not to miss such an event again.

\section{Summary}

Her X-1 has been well monitored in the past decade mostly by NuSTAR, but also by INTEGRAL and Swift/BAT, and more recently, by Insight-HXMT (Xiao et al. 2019) and Astrosat (Bala et al. 2020). Her X-1 is the only highly magnetized accreting pulsar for which repeated observations over longer periods of time exist. This has provided the base for the discovery of new phenomena, such as the dependence of the cyclotron line energy (as well as almost all spectral parameters) on flux and the long-

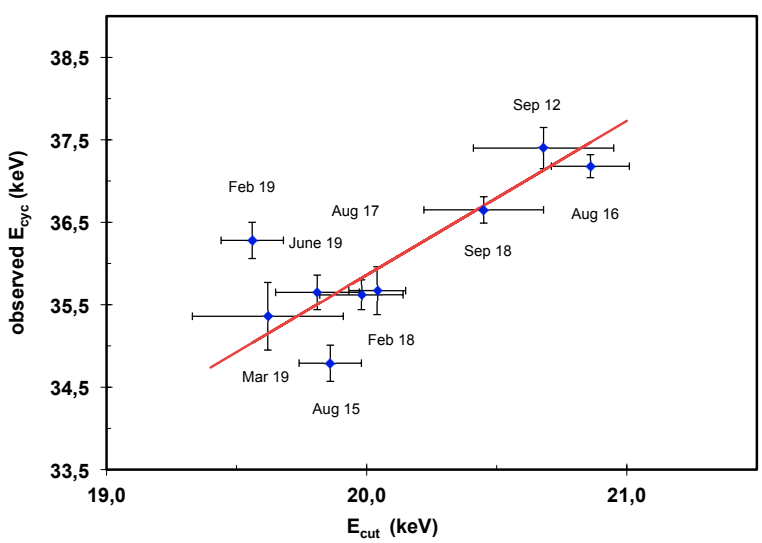

Fig. 12. Observed cyclotron line energy vs. $E_{\text {cut }}$. The best-fit line is given by the function $E_{\mathrm{cyc}}=(35.86 \pm 0.11)+(1.87 \pm 0.36) \times\left(E_{\mathrm{cut}}-20.0\right)$ (all values in $\mathrm{keV}$ ). The Pearson linear correlation coefficient is 0.79 .

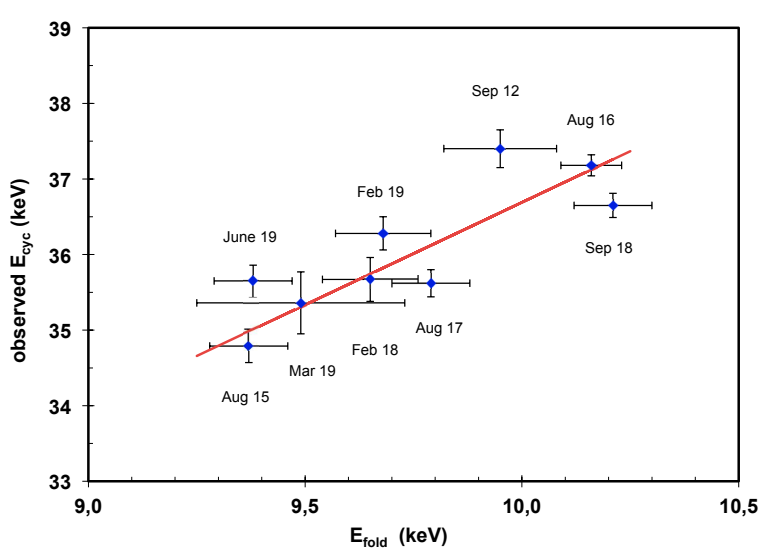

Fig. 13. Observed cyclotron line energy vs. $E_{\text {fold }}$. The best-fit line is given by the function $E_{\text {cyc }}=(36.69 \pm 0.13)+(2.71 \pm 0.39) \times\left(E_{\text {fold }}-10.0\right)$ (all values in $\mathrm{keV})$. The Pearson linear correlation coefficient is 0.82 .

term decay of the cyclotron line energy over nearly 20 years. Both of these phenomena have meanwhile been seen in other accreting X-ray binary pulsars (see the review by Staubert et al. 2019). The results of nine NuSTAR observations of Her X-1 between 2012 and 2019 are listed below.

- The dependence of the cyclotron line energy on X-ray flux, discovered in 2007, is confirmed and measured with high precision.

- The flux-normalized cyclotron line energy has been constant since (at least) $\sim 2012$. The previously reported long-term decay has ended.

- All cyclotron line parameters, that is, the line energy $E_{\text {cyc }}$, the width $\sigma$, the strength, the optical depth $\tau$, and the relative width, show a positive and linear correlation to X-ray flux.

- This statement also means that all cyclotron line parameters correlate positively and linearly with one another.

- The continuum parameters $E_{\text {cut }}$ and $E_{\text {fold }}$ correlate positively and linearly with $\mathrm{X}$-ray flux. The flux-normalized continuum parameters have been consistent with constant values since 2012. The third continuum parameter, the power-law index $\Gamma$, shows a weak positive correlation with flux. This is the opposite of what has been seen before in a pulse-amplitude-resolved analysis, which may have a somewhat different meaning. The interpretation of the different observed correlations with regard to the prevailing accretion regimes (sub- or supercritical) is not so simple. 


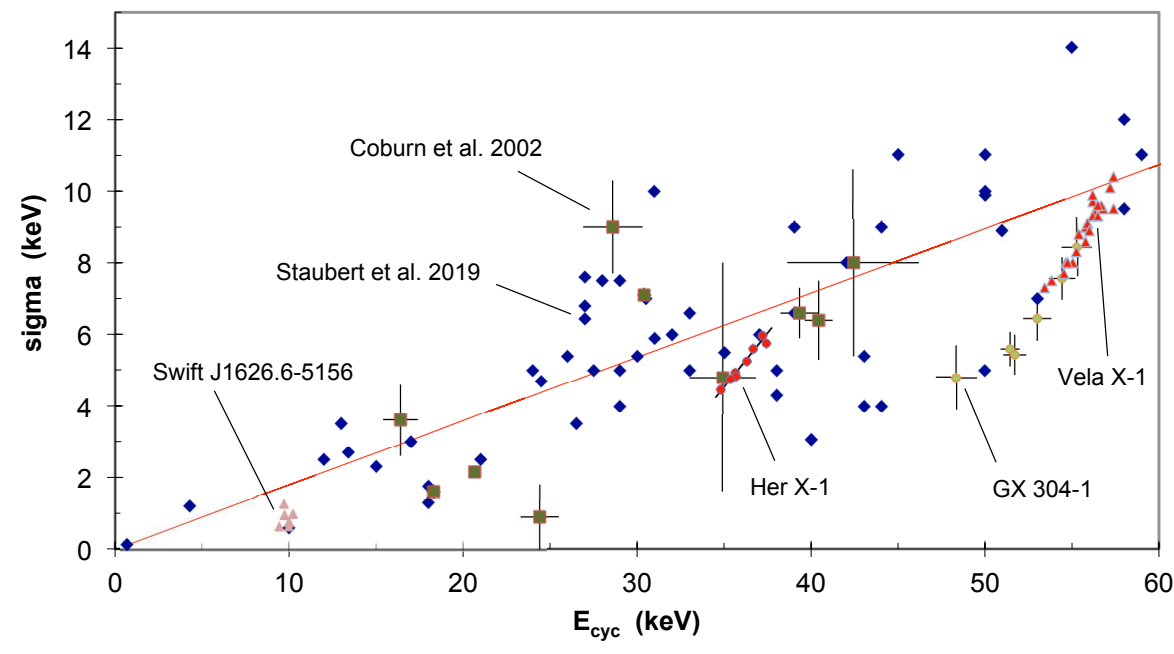

Fig. 14. Correlation between the width (sigma) and the centroid energy of cyclotron lines for different X-ray binaries. The blue rhombs are data from the compilation in Staubert et al. (2019), the filled squares (with uncertainties) are from Coburn et al. (2002). The red line is a fit to these two data sets defining $\sigma=0.18 E_{\text {cyc }}$. The individual sources are GX 304-1 (Klochkov et al. 2015), Swift J1626.6-5156 (DeCesar et al. 2013), Vela X-1 (La Parola et al. 2016), and Her X-1 (same as Fig. 4).
- We have learned that there are correlations between continuum parameters and line parameters.

- The correlations of the line and continuum parameters with X-ray flux and among each other are considered to reflect true physical correlations, which have yet to be investigated and explained by theoretical modeling. As shown in the appendix, the analysis of the purely mathematical correlation between fitting parameters has led to the conclusion that the correlation is not significant.

We urge that the source continues to be observed regularly. This seems to be secured for 2020 through already planned observations (partly simultaneous) between INTEGRAL, $\mathrm{NuS}$ $T A R, X M M-N e w t o n$ and Insight-HXMT. At the same time, it would be very important that theoretical models be developed further.

Acknowledgements. We would like to acknowledge the dedication of all the people who have contributed to the great success of all relevant missions, in particular NUSTAR. We especially thank the "schedulers", foremost Karl Forster, for his efforts with respect to the non-standard scheduling of the observations of Her X-1. We acknowledge the historical contributions to the subject at hand by Dmitry Klochkov. We thank the anonymous referee for valuable comments.

\section{References}

Araya, R., \& Harding, A. 1999, ApJ, 517, 334

Bala, S., Bhattacharya, D., Staubert, R., \& Maitra, C. 2020, MNRAS, 497, 1029

Becker, P. A., Klochkov, D., Schönherr, G., et al. 2012, A\&A, 544, A123

Brumback, M. C., Hickox, R. C., Fürst, F. S., et al. 2020, ApJ, submitted

Caballero, I., \& Wilms, J. 2012, Mem. Soc. Astron. It., 83, 230

Coburn, W., Heindl, W. A., Rothschild, R. E., et al. 2002, ApJ, 580, 394

DeCesar, M. E., Boyd, P. T., Pottschmidt, K., et al. 2013, ApJ, 762, 61

Foreman-Mackey, D., Hogg, D. W., Lang, D., \& Goodman, J. 2013, PASP, 125, 306

Fürst, F., Grefenstette, B. W., Staubert, R., et al. 2013, ApJ, 779, 69

Gerend, D., \& Boynton, P. E. 1976, ApJ, 209, 562

Gruber, D. E., Heindl, W. A., Rothschild, R. E., et al. 2001, ApJ, 562, 499

Harrison, F. A., Craig, W. W., Christensen, F. E., et al. 2013, ApJ, 770, 103

Heindl, W. A., Rothschild, R. E., Coburn, W., et al. 2004, in X-ray Timing 2003:

Rossi and Beyond, eds. P. Kaaret, F. K. Lamb, \& J. H. Swank, AIP Conf. Ser., 714,323

Isenberg, M., Lamb, D. Q., \& Wang, J. C. L. 1998, ApJ, 493, 154

Ji, L., Staubert, R., Ducci, L., et al. 2019, MNRAS, 484, 3797

Klochkov, D. K., Shakura, N. I., Postnov, K. A., et al. 2006, Astron. Lett., 32, 804

Klochkov, D., Staubert, R., Postnov, K., et al. 2008, A\&A, 482, 907

Klochkov, D., Staubert, R., Santangelo, A., Rothschild, R. E., \& Ferrigno, C. 2011, A\&A, 532, A126

Klochkov, D., Staubert, R., Postnov, K., et al. 2015, A\&A, 578, A88

Kuster, M., Wilms, J., Staubert, R., et al. 2005, A\&A, 443, 753
La Parola, V., Cusumano, G., Segreto, A., \& D’Aì, A. 2016, MNRAS, 463, 185 Makishima, K., \& Mihara, T. 1992, Frontiers O X-ray Astronomy (Tokyo: Universal Academy Press Inc.), 23

Makishima, K., Mihara, T., Nagase, F., \& Tanaka, Y. 1999, ApJ, 525, 978

Malacaria, C., Klochkov, D., Santangelo, A., \& Staubert, R. 2015, A\&A, 581, A121

Meszaros, P., \& Nagel, W. 1985, ApJ, 298, 147

Mihara, T., Ohashi, T., Makishima, K., et al. 1991, PASJ, 43, 501

Mukherjee, D., \& Bhattacharya, D. 2012, MNRAS, 420, 720

Mukherjee, D., Bhattacharya, D., \& Mignone, A. 2013, MNRAS, 435, 718

Mukherjee, D., Bhattacharya, D., \& Mignone, A. 2014, Eur. Phys. J. Web Conf., 64, 2004

Mushtukov, A. A., Suleimanov, V. F., Tsygankov, S. S., \& Poutanen, J. 2015, MNRAS, 447, 1847

Orlandini, M., Dal Fiume, D., Frontera, F., et al. 1998, ApJ, 500, L163

Parmar, A. N., Sanford, P. W., \& Fabian, A. C. 1980, MNRAS, 192, 311

Petterson, J. A. 1977, ApJ, 218, 783

Postnov, K., Shakura, N., Staubert, R., et al. 2013, MNRAS, 435, 1147

Postnov, K. A., Gornostaev, M. I., Klochkov, D., et al. 2015, MNRAS, 452, 1601

Revnivtsev, M., \& Mereghetti, S. 2016, Magnetic Fields of Neutron Stars in X-ray Binaries (Berlin, Germany: Springer), 54, 299

Reynolds, A. P., Quaintrell, H., Still, M. D., et al. 1997, MNRAS, 288, 43

Rodes-Roca, J., Torrejón, J. M., \& Bernabéu, J. G. 2008, The Fundamental Cyclotron Line in 4U 1538-52, 3, 189

Rothschild, R. E., Kühnel, M., Pottschmidt, K., et al. 2017, MNRAS, 466, 2752

Sazonov, S., Paizis, A., Bazzano, A., et al. 2020, New Astron. Rev., 88, 101536

Schandl, S., \& Meyer, F. 1994, A\&A, 289, 149

Schönherr, G., Wilms, J., Kretschmar, P., et al. 2007, A\&A, 472, 353

Schwarm, F.-W., Schönherr, G., Falkner, S., et al. 2017, A\&A, 597, A3

Staubert, R. 2003, in Multifrequency Behaviour of High Energy Cosmic Sources, ed. L. S. G. F. Giovanelli, ChJAA, 3, S270

Staubert, R. 2014, Proc. of Science (Integral 2014), 24

Staubert, R., Bezler, M., \& Kendziorra, E. 1983, A\&A, 117, 215

Staubert, R., Shakura, N. I., Postnov, K., et al. 2007, A\&A, 465, L25

Staubert, R., Klochkov, D., Vasco, D., et al. 2013, A\&A, 550, A110

Staubert, R., Klochkov, D., Wilms, J., et al. 2014, A\&A, 572, A119

Staubert, R., Klochkov, D., Vybornov, V., Wilms, J., \& Harrison, F. A. 2016, A\&A, 590, A91

Staubert, R., Klochkov, D., Fürst, F., et al. 2017, A\&A, 606, L13

Staubert, R., Trümper, J., Kendziorra, E., et al. 2019, A\&A, 622, A61

Tananbaum, H., Gursky, H., Kellogg, E. M., et al. 1972, ApJ, 174, L143

Terada, Y., Mihara, T., Nagase, F., et al. 2007, Adv. Space Res., 40, 1485

Trümper, J., Pietsch, W., Reppin, C., et al. 1977, Annal. New York Acad. Sci., 302,538

Trümper, J., Pietsch, W., Reppin, C., et al. 1978, ApJ, 219, L105

Trümper, J., Kahabka, P., Oegelman, H., Pietsch, W., \& Voges, W. 1986, ApJ, 300, L63

Vasco, D. 2012, PhD Thesis, Univ. of Tübingen, Germany

Vasco, D., Staubert, R., Klochkov, D., et al. 2013, A\&A, 550, A111

Voges, W., Pietsch, W., Reppin, C., et al. 1982, ApJ, 263, 803

Vybornov, V., Klochkov, D., Gornostaev, M., et al. 2017, A\&A, 601, A126

Wilms, J. 2012, Proceed. 39th COSPAR Sci. Assembly, 14-22 July 2012, Mysore, India, 39, 2159

Wolff, M. T., Becker, P. A., Gottlieb, A. M., et al. 2016, ApJ, 831, 194

Xiao, G. C., Ji, L., Staubert, R., et al. 2019, J. High Energy Astrophys., 23, 29 


\section{Appendix A: Correlations between spectral parameters related to the fitting process}

In the main text, we reported the correlations between spectral parameters observed from nine NUSTAR observations in 2012-2019. Here we determine the contribution of model degeneracies during the spectral fitting to theses correlations. We investigate this through Monte Carlo simulations. In practice, we adopted the best-fitting parameters of the NuSTAR observation in September 2018 as a reference model (with a flux of 5.6 (ASM-cts s ${ }^{-1}$ ), this observation is close to the center of the flux range encountered between 2012 and 2019). With the statistics and the spectral parameters from this observation as an input model, we performed two different simulations (with $10^{4}$ events each). First, an MCMC simulation (provided by $\mathrm{XSPEC}^{12}$ ), which made use of the Goodman-Weare algorithm ${ }^{13}$ (see also Foreman-Mackey et al. 2013), and second, producing simulated spectra using the fakeit command in XSPEC; these spectra were subsequently fit. The two methods provided consistent results. We show the MCMC simulation in Fig. A.3.

As expected, most of the correlations between the parameters are weak, as is evident from the circular shape of the twodimensional distributions. As an example, we show the scatter plot of $\sigma_{\text {cyc }}$ versus $E_{\text {cyc }}$ of the first 1000 simulated MCMC spectra in Fig. A.1. The range of observed $E_{\text {cyc }}$ is a factor $\sim 10$ larger than the corresponding FWHM of the simulated $E_{\text {cyc }}$ distribution (for $\sigma_{\text {cyc }}$, the factor is $\sim 3.3$ ). This shows that any model degeneracy contributes only little to the overall correlation. Three distributions are elongated (under roughly $45^{\circ}$ ), indicating stronger correlations: $D_{\text {cyc }}{ }^{14}$ versus $\sigma_{\text {cyc }}, E_{\text {cut }}$ versus $\sigma_{\text {cyc }}$, and $E_{\text {cut }}$ versus $D_{\text {cyc. }}$. Even here the corresponding factors (FWHM/observed range) are between two and four. The correlation between $\sigma_{\text {cyc }}$ and $D_{\text {cyc }}$ is given by the definition $D_{\text {cyc }}=\sigma \times \tau \times \sqrt{2 \pi}$. In Fig. A.2 we show the degeneracy between the continuum parameter $E_{\text {cut }}$ and the line parameter $\sigma_{\text {cyc }}$. We conclude that the discussed physical correlations are real.

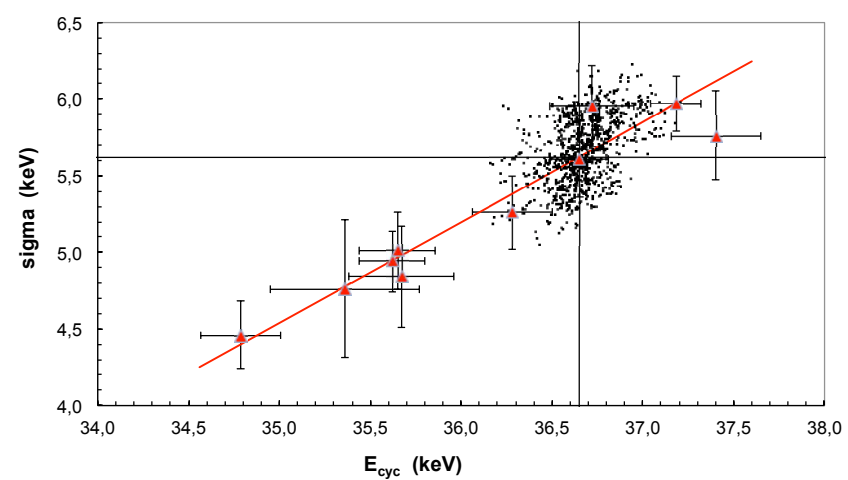

Fig. A.1. First $1000 \mathrm{sigma} / E_{\text {cyc }}$ pairs of the MCMC simulation together with the measured correlation as shown in Fig. 4.

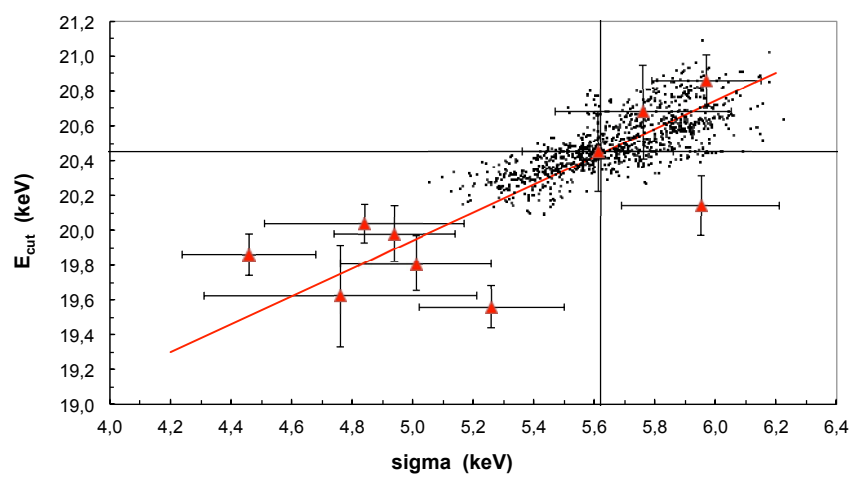

Fig. A.2. First $1000 E_{\text {cut }} /$ sigma pairs of the MCMC simulation together with the measured correlation.

\footnotetext{
12 https://heasarc.gsfc.nasa.gov/xanadu/xspec/

13 https://ui . adsabs . harvard . edu/abs/2010CAMCS...5 ... 65G/abstract

${ }^{14} D_{\text {cyc }}$ is called strength in the main text.
} 


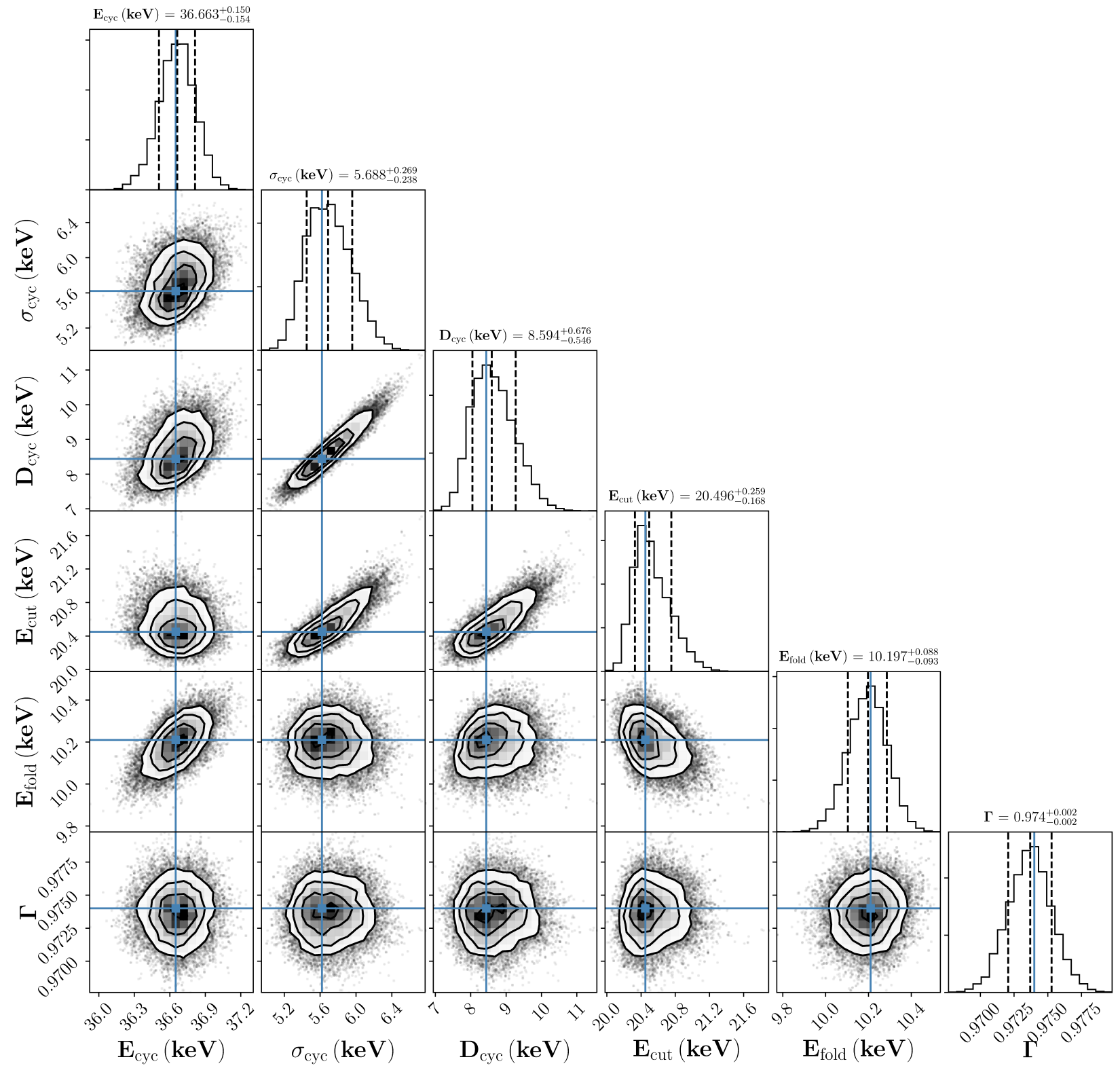

Fig. A.3. Contours represent two-dimensional distributions of parameters obtained from Monte Carlo simulations at the significance level of 1, 2, and $3 \sigma$, and the histograms are distributions for each variable. The blue lines are the input parameters we used in the simulations. 